\title{
THE MICROARCSECOND QUASAR J1819+3845: POLARIZATION OBSERVATIONS AND DETAILED LIGHTCURVE ANALYSIS
}

\author{
A.G. DE BRUYN and J. DENNETT-THORPE
}

ASTRON, Dwingeloo and Kapteyn Institute, Groningen

\begin{abstract}
The quasar $\mathbf{J} 1819+3845$ shows exceedingly rapid variations in both total intensity and linear polarization. These are attributed to interstellar scintillation. The source contains at least two structural components each of which shows variable polarization behaviour. The polarization variations are generally, but perhaps not always, coherent over the $80 \mathrm{MHz}$ band setting. The $6 \mathrm{~cm}$ lightcurves in the first few months of 2000 hint at a time-asymmetric behaviour as could be expected from an asymmetric source structure.
\end{abstract}

\section{Introduction}

The quasar $\mathrm{J} 1819+3845$ is the most rapidly variable extragalactic radio source in the sky; these variations are due to interstellar scintillation (Dennett-Thorpe and De Bruyn, 2000). In a companion paper in these proceedings we presented the results of a full year of monitoring with the Westerbork Synthesis Radio Telescope at a frequency of $5 \mathrm{GHz}$. Here we present a high resolution lightcurve taken from this database and discuss preliminary results of variable linear polarization. We also draw attention to peculiar features in the lightcurve suggestive of a time-asymmetric behaviour.

\section{Polarization Variations}

$\mathrm{J} 1819+3845$ was observed regularly, for a total time of about $12 \mathrm{~h}$ per month, since the discovery observations of May 1999. The May 1999 data did not have sufficient sensitivity to detect or analyse the linear polarization (they were taken with a still incomplete array split to observe two frequencies simultaneously). The new data reported here have about 3 times better sensitivity, with a noise level of $0.5 \mathrm{mJy}$ in Stokes $I$ and $Q$ and $0.7 \mathrm{mJy}$ in Stokes $U$ and $V$ after 2 minutes of integration. The difference in noise levels in the various Stokes parameters is related to details of the continuum backend hardware. All data were taken in 8 adjacent, independently processed, $10 \mathrm{MHz}$ bands (see Dennett-Thorpe and De Bruyn, 2000, for more details). The linearly polarized signal of $\mathrm{J} 1819+3845$ rarely exceeds $1-2 \mathrm{mJy}$, which is about $1 \%$ of the average total flux density of $150 \mathrm{mJy}$. 


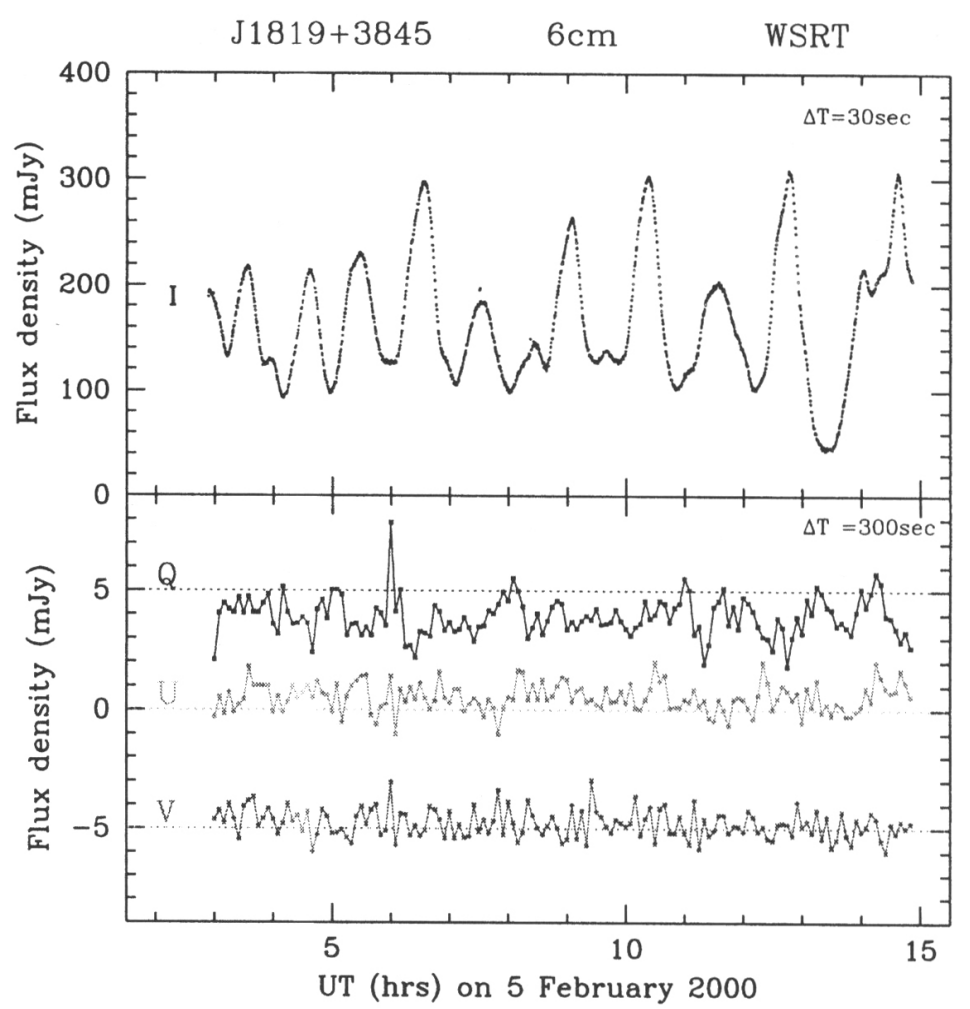

Figure 1. Fast variations in the quasar J1819+3845 at $6 \mathrm{~cm}$. Time resolution in Stokes $I$ is 30 seconds, in $Q, U$ and $V 5$ minutes. Note that the polarization lightcurves are shown on a more than $20 \times$ larger scale. The dotted lines indicate the zero level of the corresponding curve.

We have not detected any believable circular polarization. The typical instrumental errors in the polarization are $0.1 \%(1 \sigma)$ of Stokes $I$ as deduced from observations of pairs of strong polarized and unpolarized calibrators observed before and after the usually uninterrupted $12 \mathrm{~h}$ runs.

The low percentage polarization of the source complicates the polarization analysis. This is exacerbated by the presence of several distinct structural components in the source. As discussed in Dennett-Thorpe and De Bruyn (2000) there appears to be a base-level flux density (about 50-70 mJy) below which the source intensity never goes, in spite of the strong modulations (see also the companion paper in these proceedings). The rapid scintillations of J1819+3845 should allow, in principle, to disentangle the relative polarization characteristics of these two components.

The analysis of the polarization data is still in progress, hence we can only provide some very preliminary results and conclusions. It is clear that both components have different polarization, both of are intrinsically variable on a timescale 
of months, i.e. much longer than 12 hours. The polarization of $\mathbf{J} 1819+3845$ reveals a number of features reminiscent of those in the quasar B0917+624 (Rickett et al., 1995). Figure 1 shows the lightcurve of 5 February 2000. We will first describe the polarization, drawing from several lightcurves, but using this one as an example. Some details on the total intensity behaviour will be discussed in the next section.

To improve the $\mathrm{S} / \mathrm{N}$ in the polarized signal the data were averaged over 5 minutes. The data have been analysed in a variety of ways. Images made from the full 12 hour runs have a noise level of about $40 \mu \mathrm{Jy}$ and allow an accurate measurement of the time-averaged polarization, which is found to vary over the year. However, the 12-hour images are a composite of signals from the 'extended' component and the compact source which, once averaged over 12 hours, have similar flux densities of about 60-70 mJy and 70-80 mJy, respectively. In general the polarization appears to be the same to within the noise in each of the eight $10 \mathrm{MHz}$ bands. Because the polarization is best seen in the compact component, which frequently goes up to $250 \mathrm{mJy}$, we can set an upper limit to its RM of a few thousand $\mathrm{rad} \mathrm{m}^{-2}$. However, in at least one of the runs we found a systematic $20^{\circ}$ rotation in the polarization angle across the band which would imply a RM of about $4000 \mathrm{rad} \mathrm{m}^{-2}$. On at least one occasion there also appeared to be a drift in the polarization level during 12 hours. If real, the interpretation of this is not straightforward. If the two components have very different RMs their contribution to the polarization vector will change with frequency during scintillations.

A detailed inspection of the lightcurves in Figure 1 reveals that especially the Stokes $Q$ signal oscillates with total intensity, but often out of phase. This suggests polarization sub-structure in the compact component. Both the base-level and compact component polarization signals are found to vary on a timescale of months. Stokes $Q$, for which we have about 1.4 times better $\mathrm{S} / \mathrm{N}$ than Stokes $U$, has shown several sign reversals in the last year. A more complete analysis of the polarization will be presented at a later date.

\section{Time-Asymmetry in the Lightcurves?}

The lightcurves of 30 December 1999 (both 3.6 and $6 \mathrm{~cm}$ ) and 21 January 2000 hinted at a rather interesting feature in the lightcurves: the highest peaks in the lightcurve are asymmetric with a slower rise than fall. This is particularly noticeable in the high time resolution lightcurves. This asymmetry persists in the February 52000 lightcurve shown in Figure 1 and can be seen best by looking at the separation of the individual data points leading up to and after the scintillation peaks (scintles). The asymmetry appears not to be significant in the $18 \mathrm{March}$ 2000 data. Assuming the apparent asymmetries survive appropriate statistical tests, could they be related to source structure? The intensity pattern projected onto the solar system is the convolution of the intrinsic source intensity distribution with the point source scintillation pattern (Salpeter, 1967). The current size upper limit 
on the source (about 30 microarcseconds, the $5 \mathrm{GHz}$ Fresnel scale for a screen at a distance of 20 parsecs; Dennett-Thorpe and De Bruyn, 2000) assumes a spherically symmetric source. If $\mathrm{J} 1819+3845$ is asymmetric on this angular scale, we should see a systematic change in the lightcurve as the earth moves through the scintles.

When the effective velocity vector of the earth rotates, during the year, relative to the projected source asymmetry direction, the situation changes. For a stable source structure the asymmetry could then be absent for many months to reappear after about 6 months with opposite sign. In order for the effect to be detectable above the noise we need a large number of scintles. Unfortunately the scintillations in July-September 1999 are so much slower, due to the effect that the effective velocity goes to almost zero, we cannot test this prediction with this data.

If the source structure remains unchanged for a whole year, and that is a very big IF for a source with a maximum dimension of only a few lightmonths expanding at presumably highly super-luminal speeds, it will also influence the seasonal variation of the scintillation timescale as discussed in the companion paper in these proceedings. There it was concluded that there is no evidence for a 6 month periodicity.

\section{References}

Dennett-Thorpe, J. and de Bruyn, A.G.: 2000, The discovery of a microarcsecond quasar: J1819+3845, Astrophys. J. Lett. 529, 65-68.

Rickett, B.J., Quirrenbach, A., Wegner, R., Krichbaum, T. and Witzel, A.: 1995, Interstellar scintillation of the Radio Source 0917+624, Astron. Astrophys. 293, 479-492.

Salpeter, E.E.: 1967, Interstellar scintillation. I. Theory, Astrophys. J. 147, 433. 